\title{
The Essential Guide to Image Processing
}

Al Bovik, Ed., 672 pp., ISBN: 978-0123744579, Academic Press (2009), \$89.95 paperback.

Reviewed by James E. Adams, Jr., Kodak Research Labs, Eastman Kodak Company, Rochester, New York.

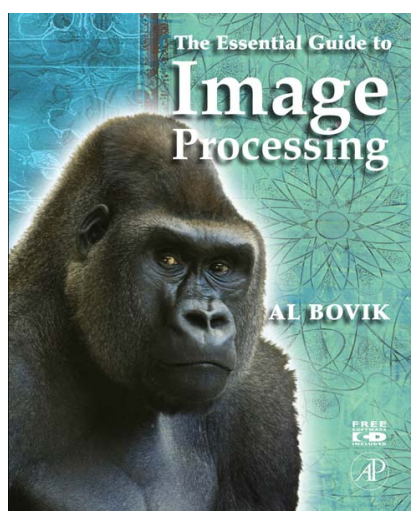

The Essential Guide to Image Processing is the latest incarnation of $\mathrm{Al}$ Bovik's wellknown Handbook of Image and Video Processing. The Guide is a collection of chapters written by a variety of experts to provide, as stated in the preface, a "highly accessible" entry into the field of "introductory, intermediate, and advanced topics of digital image processing." The first five chapters are tutorials on basic image-processing operations, such as histogram stretching, bilinear interpolation resizing, binary image morphological operations, and the basics of discrete Fourier transform operations. These are followed by the heart of the book: sixteen chapters on a variety of topics ranging from the broad scopes of wavelet analysis and nonlinear filtering techniques (e.g., median filtering and wavelet denoising) to more specific discussions of JPEG and JPEG2000 and partial differential equation iterative processes. The book concludes with six exciting chapters that pull together many of the tools discussed earlier in the book to address a number of topics of current research and application, such as image watermarking, face and iris recognition, and computed tomography.

For those familiar with the Handbook, the Guide's major departure is the relocation of all video-related image processing topics into a separate text, The Essential Guide to Video Processing. Bovik quips in the preface why the bifurcation: "While this previous book [Handbook] was very well-received, its sheer size made it highly un-portable (but a fantastic doorstop)." As one who appreciates having the unified Handbook on my shelf with both static and motion-related topics in one package, the lack of motion-related discussions in the present imageprocessing Guide is vaguely disappointing, even if the obvious solution is to also get a copy of the video Guide! Another addition to the Guide is a CD with the signal, image, and video audiovisualization (SIVA) educational courseware, which is written in LabVIEW. (A standalone LabVIEW environment is also provided.) Through a simple GUI, this software allows one to perform some of the more basic image-processing operations (e.g., thresholding) on a library of supplied images and to see the results in real time. This software will be discussed in more detail below.

The Guide begins with chapters defining an image and introducing the SIVA software. The book really begins to gain traction with Chapter 3 on basic gray-level image-processing techniques. Histogram manipulation (linear and nonlinear), image arithmetic (e.g., adding and subtracting two images), and affine transform operations are all presented with enough details and illustrations that the reader can immediately start implementing these techniques in their own image-processing code. The next chapter on basic binary image processing quickly gets into interesting topics such as region labeling and subsequent region processing through morphological operations. Again, the reader is given all the details necessary for immediate application to their own image-processing activities. The tutorial section of the book concludes with a chapter on basic Fourier analysis. This could be a significantly more challenging presentation for the classroom student with the introduction of topics such as cyclic versus linear convolution and all the associated ramifications. Bovik, who pens this chapter himself, does an excellent job of keeping the discussion simple, succinct, and methodical to limit the number of subtleties the reader has to digest at any given time.

Upon leaving the tutorial section, the manner of presentation starts to vary significantly from chapter to chapter. This seems to be driven more by the individual author writing styles and approaches than by the complexity of the topics itself. After the explicit "here's how you do it" presentations of the tutorial section, the next chapter on wavelet decomposition is less satisfying. All the important concepts, terminology, and strategic approaches to wavelet decomposition are presented in a clear and accessible manner. By the time the reader is finished with the chapter, he or she will be able to enter the vast body of literature on this topic and make knowledgeable searches for the information he or she may truly want. However, readers may have to search external references if they have the task of actually implementing wavelet decomposition; e.g., the discussion of using the classic 4-tap Daubechies wavelet filter to decompose an image may be too succinct for a reader just entering the field.

The remaining chapters in the book oscillate about this point of narrowing the focus in order to provide enough detail to actually implement the technology, versus reducing such detail in favor of expanding the breadth of coverage in order to provide a more comprehensive perspective of the field. After wavelet decomposition, one encounters chapters on image noise models, color science á là Joel Trussell's well-known linear-algebra approach, probabilistic image modeling, linear and nonlinear filtering and denoising, and morphological filtering. The material becomes a bit more advanced with the next two chapters on deconvolution through both direct and iterative methods. Three chapters on compression, such as the aforementioned discussion of JPEG and JPEG2000, appear next. Finally, chapters on edge detection, partial-differential equation diffusion, and image-quality assessment complete the central portion of the book.

The culmination of all this spade work is reached in the final six chapters. With the exception of the iris-recognition chapter, these chapters are more literature survey and image-capture instrumentation descriptions than detailed image-processing algorithm presentations. Much, if not most, of the details of the image-processing tasks are left to the cited references. This is not necessarily a surprise considering the complexity of the topics discussed. The first such chapter on image watermarking provides an interesting back story to the topic which was first considered to be a relatively straightforward task until all the subtleties of the problem were truly appreciated. As a result, 
instead of becoming yet another triumph in image-processing research, watermarking is still a problem looking for an attractive solution. Chapters on automatic fingerprint recognition and unconstrained face recognition follow next. The aforementioned chapter on iris recognition is next in line and has a distinctly different flavor from the other chapters in this section. Unlike the other topics which still seem to be in the process of maturing, iris detection is digested and boiled down to its key essence and presented in a simple and straightforward manner, such that today's active research topics are tomorrow's classroom textbook material. Finally, chapters on computed tomography and computer-assisted microscopy are presented. Each of these chapters has at least as much to say about the equipment and means of capturing the raw image data as they do about the subsequent image processing of the results.

At the back of the book one finds the CD with the SIVA software. This software installs easily enough and is fairly selfevident to run with a nice contextual help window wherever the mouse is pointing. The image-processing operations illustrated are largely the basic ones presented in the tutorial section with a few extras added. The software seemed fairly robust, though the first time I ran the DFT option on the cameraman scene I was bemused to be looking at the exact same (an incorrect) image in both the magnitude and phase windows. Rerunning the program from scratch fixed that problem. The LiveVIEW source code is provided, but without a full LiveVIEW environment on my computer, I was unable to view the source code.

Bovik describes the intended scope of this book as ranging from a "classroom textbook" to a "reference for the practicing image-processing engineer, scientist, or researcher."
The Essential Guide to Image Processing succeeds admirably in providing something for everyone, though it also carries the consequence that not all chapters will be valued by all readers. The classroom student will probably find some chapters provide too dense a presentation of the material to be readily accessible, while researchers in the field may pass over some chapters for having too basic an approach that stops just when things are getting interesting! Fortunately, in both situations each chapter is heavily referenced with important and relevant sources that can be used to find both more fundamental and more detailed discussions of the topic in question.

The only quibble I have with the Guide is the complete lack of color images and illustrations. More precisely, a number of chapters refer to colors within the cited figures and images that are clearly grayscale renderings of what were originally color figures and images. Sometimes it is evident what the author is referring to, but not always. This was addressed in the Handbook by a number of color plates. The color plates are gone from the Guide, but the corresponding text has not been updated. This is the only disconnect in an otherwise excellent book!

James E. Adams, Jr., is a senior principal scientist at Kodak Research Labs. He holds 34 patents in the field of digital image processing, most notably in the fields of color filter array interpolation and noise reduction. He is a Kodak Distinguished Inventor and 2005 RIPLA Inventor of the Year. He has authored several technical articles and book chapters on digital-camera image processing. 